\title{
Massive Dirac fermions and spin physics in an ultrathin film of topological insulator
}

\author{
Hai-Zhou Lu, ${ }^{1}$ Wen-Yu Shan, ${ }^{1}$ Wang Yao, ${ }^{1}$ Qian Niu, ${ }^{2}$ and Shun-Qing Shen ${ }^{1}$ \\ ${ }^{1}$ Department of Physics and Center for Theoretical and Computational Physics, The University of Hong Kong, Pokfulam Road, \\ Hong Kong, China \\ ${ }^{2}$ Department of Physics, The University of Texas, Austin, Texas 78712-0264, USA \\ (Received 14 August 2009; revised manuscript received 26 January 2010; published 3 March 2010)
}

\begin{abstract}
We study transport and optical properties of the surface states, which lie in the bulk energy gap of a thin-film topological insulator. When the film thickness is comparable with the surface-state decay length into the bulk, the tunneling between the top and bottom surfaces opens an energy gap and form two degenerate massive Dirac hyperbolas. Spin-dependent physics emerges in the surface bands, which are vastly different from the bulk behavior. These include the surface spin Hall effects, spin-dependent orbital magnetic moment, and spin-dependent optical transition selection rule, which allows optical spin injection. We show a topological quantum phase transition where the Chern number of the surface bands changes when varying the thickness of the thin film.
\end{abstract}

DOI: 10.1103/PhysRevB.81.115407

PACS number(s): 72.25.-b, 78.67.-n, 85.75.-d

\section{INTRODUCTION}

A three-dimensional (3D) topological insulator is a novel quantum state of matter, which possesses metallic surface states in the bulk energy gap. ${ }^{1-4}$ The surface states consist of an odd number of massless Dirac cones, which are protected by $Z_{2}$ topological invariants. The first 3D topological insulator is $\mathrm{Bi}_{x} \mathrm{Sb}_{1-x}$, an alloy with complex structure of surface states, which was confirmed by using angle-resolved photoemission spectroscopy. ${ }^{5-7}$ Recently it was verified that $\mathrm{Bi}_{2} \mathrm{Se}_{3}$ and $\mathrm{Bi}_{2} \mathrm{Te}_{3}$ have only one Dirac cone near the $\Gamma$ point by both experiments and the first-principles calculation, ${ }^{8-11}$ which attracts extensive attentions in condensed matter physics. The electrons or Dirac fermions in the surface states of topological insulator obey the $2+1$ Dirac equations and reveal a lot of unconventional properties such as the topological magnetoelectric effect. ${ }^{12}$ It was also proposed that the surface states interfaced with a superconductor can form Majorana fermions for performing fault-tolerant quantum computation. ${ }^{13-18}$ Since the surface states surround the sample, it is still a great challenge for both experimentalists and theorists to explore the transport properties for metallic surface states in the topological insulators.

In this paper, we study an ultrathin film of topological insulator where tunneling between the surface states on the top and bottom surfaces opens a finite gap in the Dirac cone centered at the $\Gamma$ point $(k=0)$. The low-lying physics of the ultrathin film can be described as two degenerate massive Dirac hyperbolas, which form time-reversal copy of each other. Each massive band has a k-dependent spin configuration: one near the $\Gamma$ point determined by the energy gap and the other at $k$ large enough determined by a spin-orbit coupling term quadratic in $k$. We show that the energy gap oscillates with the thin-film thickness, and changes sign at critical thicknesses. Across the transition points, the $\mathbf{k}$-dependent spin configuration near the $\Gamma$ point is flipped while those at large $k$ remains unchanged, leading to a topological quantum phase transition where the Chern numbers of the surface bands change [Eq. (31)]. In the two Dirac hyperbolas of opposite spin configurations, the $\mathbf{k}$-dependent spin structure re- sults in a distribution of orbital magnetic moment and Berry curvature with opposite signs. In doped metallic regime, the Berry curvature drives the spin Hall effect of the extra carriers, which leads to net spin accumulations on the thin-film edges. We also discover a spin-dependent optical transition selection rule, which allows optical injection of spins in the thin film.

The paper is organized as follows. In Sec. II, we present the derivation of the effective Hamiltonian for the thin film of topological insulator. In Sec. III, the oscillation of the gap and the $\mathbf{k}$-dependent spin configuration are discussed. In Sec. $\mathrm{IV}$, the orbital magnetic moment and Berry curvature are addressed. In Sec. V, the spin Hall conductance is derived in detail. In Sec. VI, the spin-dependent optical transition selection rule is shown. Finally, a conclusion is given in Sec. VII.

\section{MODEL FOR AN ULTRATHIN FILM}

We start with the low-lying effective model for bulk $\mathrm{Bi}_{2} \mathrm{Se}_{3}$, in which surface states consist of a single Dirac cone at the $\Gamma$ point. ${ }^{9}$ We take the periodic boundary conditions in the $x-y$ plane such that $k_{x}$ and $k_{y}$ are good quantum numbers, and denote the thickness of the thin film along $z$ direction as $L$. On the basis of $\left\{\left|p 1_{z}^{+}, \uparrow\right\rangle,\left|p 2_{z}^{-}, \uparrow\right\rangle,\left|p 1_{z}^{+}, \downarrow\right\rangle,\left|p 2_{z}^{-}, \downarrow\right\rangle\right\}$, which are the hybridized states of Se $p$ orbital and Bi $p$ orbital, with even $(+)$ and odd $(-)$ parities, the model Hamiltonian is given by

$$
H(k)=\left(C-D_{1} \partial_{z}^{2}+D_{2} k^{2}\right)+\left(\begin{array}{cc}
h\left(A_{1}\right) & A_{2} k_{-} \sigma_{x} \\
A_{2} k_{+} \sigma_{x} & h\left(-A_{1}\right)
\end{array}\right),
$$

where

$$
h\left(A_{1}\right)=\left(M+B_{1} \partial_{z}^{2}-B_{2} k^{2}\right) \sigma_{z}-i A_{1} \partial_{z} \sigma_{x}
$$

and $\sigma_{\alpha}$ are the Pauli matrices with $k_{ \pm}=k_{x} \pm i k_{y}$ and $k^{2}=k_{x}^{2}+k_{y}^{2}$. This model is invariant under time-reversal symmetry and inversion symmetry. In this paper, the model parameters are adopted from Ref. 9: $M=0.28 \mathrm{eV}, A_{1}$ $=2.2 \mathrm{eV} \AA, A_{2}=4.1 \mathrm{eV} \AA, B_{1}=10 \mathrm{eV} \AA^{2}, B_{2}=56.6 \mathrm{eV} \AA^{2}$, $C=-0.0068 \mathrm{eV}, \quad D_{1}=1.3 \mathrm{eV} \AA^{2}, \quad$ and $D_{2}=19.6 \mathrm{eV} \AA^{2}$. 
Though we adopt this concrete model to study the properties of an ultrathin film of topological insulator, the general conclusion in this paper should be applicable to other topological insulators.

To establish an effective model for an ultrathin film, we first find the four solutions to the surface states of the model in Eq. (1) at the $\Gamma$ point $\left(k_{x}=k_{y}=0\right),{ }^{19}$

$$
H_{0}=\left[\begin{array}{cc}
h_{0}\left(A_{1}\right) & 0 \\
0 & h_{0}\left(-A_{1}\right)
\end{array}\right]
$$

where

$$
h_{0}\left(A_{1}\right)=C-D_{1} \partial_{z}^{2}+\left(M+B_{1} \partial_{z}^{2}\right) \sigma_{z}-i A_{1} \partial_{z} \sigma_{x} .
$$

The solution of the block-diagonal $H_{0}$ can be found by putting a two-component trial solution into the eigenequation of the upper block

$$
h_{0}\left(A_{1}\right)\left(\begin{array}{l}
a \\
b
\end{array}\right) e^{\lambda z}=E\left(\begin{array}{l}
a \\
b
\end{array}\right) e^{\lambda z}
$$

with $a, b$, and $\lambda$ the trial coefficients defining the behavior of the wave functions and $E$ the trial eigenenergy. Note that the trial coefficients may have multiple solutions, the final solution should be a linear superposition of these solutions with the superposition coefficients determined by boundary conditions. Then the problem becomes a straightforward calculation of the Schrödinger equation. Considering an open boundary condition that the wave functions vanish at the two surfaces located at $z= \pm L / 2$ of the film, we finally obtain two transcendental equations

$$
\frac{\left[C-M-E-\left(D_{1}+B_{1}\right) \lambda_{1}^{2}\right] \lambda_{2}}{\left[C-M-E-\left(D_{1}+B_{1}\right) \lambda_{2}^{2}\right] \lambda_{1}}=\frac{\tanh \left(\lambda_{\alpha} L / 2\right)}{\tanh \left(\lambda_{\bar{\alpha}} L / 2\right)},
$$

note that where $\alpha=1$ and $2, \bar{\alpha}=2$ if $\alpha=1$, vice versa, so there are two transcendental equations. In Eq. (4), $\lambda_{\alpha}$ define the behavior of the wave functions along $z$ axis and are functions of the energy $E$

$$
\lambda_{\alpha}(E)=\sqrt{\frac{-F+(-1)^{\alpha-1} \sqrt{R}}{2\left(D_{1}^{2}-B_{1}^{2}\right)}}
$$

where for convenience we have defined $F=A_{1}^{2}+2 D_{1}(E-C)$ $-2 B_{1} M$ and $R=F^{2}-4\left(D_{1}^{2}-B_{1}^{2}\right)\left[(E-C)^{2}-M^{2}\right]$. The selfconsistent solution to the two equations in Eq. (4) can be found numerically and give two energies at the $\Gamma$ point, i.e., $E_{+}$and $E_{-}$, which define an energy gap

$$
\Delta \equiv E_{+}-E_{-}
$$

Note that the bulk states with much higher or lower energies due to the quantization of $k_{z}$ in the finite quantum well, in principle, can also be obtained in this way, but are ignored here because we concentrate on the surface states near the gap. The eigen wave functions for $E_{+}$and $E_{-}$are, respectively,

$$
\begin{aligned}
& \varphi^{\uparrow}\left(A_{1}\right)=C_{+}\left[\begin{array}{c}
-\left(D_{1}+B_{1}\right) \eta_{1}^{+} f_{-}^{+} \\
i A_{1} f_{+}^{+}
\end{array}\right], \\
& \chi^{\uparrow}\left(A_{1}\right)=C_{-}\left[\begin{array}{c}
-\left(D_{1}+B_{1}\right) \eta_{2}^{-} f_{+}^{-} \\
i A_{1} f_{-}^{-}
\end{array}\right],
\end{aligned}
$$

where $C_{ \pm}$are the normalization constants. The superscripts of $f_{ \pm}^{ \pm}$and $\eta_{1,2}^{ \pm}$stand for $E_{ \pm}$, and the subscripts of $f_{ \pm}^{ \pm}$for parity, respectively. The expressions for $f_{ \pm}^{ \pm}$and $\eta_{1,2}^{ \pm}$are given by

$$
\begin{gathered}
f_{+}^{ \pm}(z)=\frac{\cosh \left(\lambda_{1}^{ \pm} z\right)}{\cosh \left(\lambda_{1}^{ \pm} L / 2\right)}-\frac{\cosh \left(\lambda_{2}^{ \pm} z\right)}{\cosh \left(\lambda_{2}^{ \pm} L / 2\right)}, \\
f_{-}^{ \pm}(z)=\frac{\sinh \left(\lambda_{1}^{ \pm} z\right)}{\sinh \left(\lambda_{1}^{ \pm} L / 2\right)}-\frac{\sinh \left(\lambda_{2}^{ \pm} z\right)}{\sinh \left(\lambda_{2}^{ \pm} L / 2\right)}, \\
\eta_{1}^{ \pm}=\frac{\left(\lambda_{1}^{ \pm}\right)^{2}-\left(\lambda_{2}^{ \pm}\right)^{2}}{\lambda_{1}^{ \pm} \operatorname{coth}\left(\lambda_{1}^{ \pm} L / 2\right)-\lambda_{2}^{ \pm} \operatorname{coth}\left(\lambda_{2}^{ \pm} L / 2\right)}, \\
\eta_{2}^{ \pm}=\frac{\left(\lambda_{1}^{ \pm}\right)^{2}-\left(\lambda_{2}^{ \pm}\right)^{2}}{\lambda_{1} \tanh \left(\lambda_{1}^{ \pm} L / 2\right)-\lambda_{2}^{ \pm} \tanh \left(\lambda_{2}^{ \pm} L / 2\right)},
\end{gathered}
$$

where $\lambda_{\alpha}^{ \pm} \equiv \lambda_{\alpha}\left(E_{ \pm}\right)$can be found by putting back $E_{ \pm}$into Eq. (5).

By replacing $A_{1}$ by $-A_{1}$ in the above solutions, the energies of the lower block $h_{0}\left(-A_{1}\right)$ of $H_{0}$ are found degenerate with those of $h_{0}\left(A_{1}\right)$ and their wave functions are denoted as $\varphi^{\downarrow}\left(-A_{1}\right)$ and $\chi^{\downarrow}\left(-A_{1}\right)$, respectively. Now we have four states, namely, $\quad\left[\varphi^{\uparrow}\left(A_{1}\right), 0\right]^{T}, \quad\left[\chi^{\uparrow}\left(A_{1}\right), 0\right]^{T}, \quad\left[0, \varphi^{\downarrow}\left(-A_{1}\right)\right]^{T}, \quad$ and $\left[0, \chi^{\downarrow}\left(-A_{1}\right)\right]^{T}$, where $\uparrow$ and $\downarrow$ imply that the orbits with spin up and down are decoupled. By using these four solutions as basis states and rearranging their sequence following (note that each basis state is a four-component vector):

$$
\left(\begin{array}{c}
\varphi^{\uparrow}\left(A_{1}\right) \\
0
\end{array}\right),\left(\begin{array}{c}
0 \\
\chi^{\downarrow}\left(-A_{1}\right)
\end{array}\right),\left(\begin{array}{c}
\chi^{\uparrow}\left(A_{1}\right) \\
0
\end{array}\right),\left(\begin{array}{c}
0 \\
\varphi^{\downarrow}\left(-A_{1}\right)
\end{array}\right),
$$

we can map the original Hamiltonian to the Hilbert space spanned by these four states and reach a new low-energy effective Hamiltonian for the ultrathin film,

$$
H_{\text {eff }}=\left[\begin{array}{cc}
h_{+}(k) & 0 \\
0 & h_{-}(k)
\end{array}\right]
$$

in which

$$
h_{\tau_{z}}(k)=E_{0}-D k^{2}-\hbar v_{F}\left(k_{x} \sigma_{y}-k_{y} \sigma_{x}\right)+\tau_{z}\left(\frac{\Delta}{2}-B k^{2}\right) \sigma_{z} .
$$

Note that here the basis states of Pauli matrices stand for spin-up and spin-down states of real spin. In Eq. (11), we have introduced a hyperbola index $\tau_{z}= \pm 1$ (or \pm ). As shown in Fig. 1, one can view the hyperbolas as the $K$ and $K^{\prime}$ valleys in the staggered graphene $[(\mathrm{a})]$ but being relocated to the $\Gamma$ point $[(b)]$. Unlike the momentum correspondence in graphene, it is the $\sigma_{z}$ to $-\sigma_{z}$ correspondence in the present case. Therefore, the dispersions of $h_{ \pm}$are actually doubly 
(a)

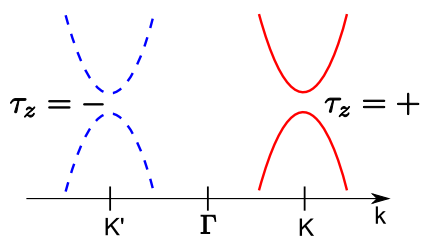

(b)

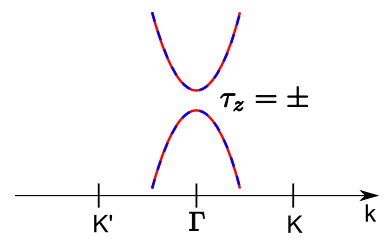

FIG. 1. (Color online) Schematic comparison between (a) the gapped $\mathrm{K}-\mathrm{K}^{\prime}$ valleys in the staggered graphene and (b) the twofold degenerate hyperbolas described by our effective Hamiltonian in Eq. (10).

degenerate, which is secured by time-reversal symmetry. Here, $\tau_{z}= \pm$ are used to distinguish the two degenerate hyperbolas, $h_{+}(k)$ and $h_{-}(k)$ describe two sets of Dirac fermions, each show a pair of conduction and valence bands with the dispersions

$$
\varepsilon_{c / v}(\mathbf{k})=E_{0}-D k^{2} \pm \sqrt{\left(\Delta / 2-B k^{2}\right)^{2}+\left(\hbar v_{F}\right)^{2} k^{2}},
$$

where $c$ and $v$ correspond to the conduction and valence bands, respectively. The eigenstates for $\varepsilon_{c / v}$ are

$$
u_{c / v}(\mathbf{k})=\frac{1}{\left\|u_{c / v}\right\|}\left[\begin{array}{c}
\left(\Delta / 2-B k^{2}\right) \tau_{z}+\varepsilon_{c / v} \\
-i \hbar v_{F} k_{+}
\end{array}\right]
$$

with $\left\|u_{c / v}\right\|=\sqrt{\left[\left(\Delta / 2-B k^{2}\right) \tau_{z}+\varepsilon_{c / v}\right]^{2}+\left(\hbar v_{F}\right)^{2} k^{2}}$. Besides the gap $\Delta$ already defined in Eq. (6), the other parameters in Hamiltonian (11) are given by

$$
\begin{gathered}
v_{F}=\left(A_{2} / \hbar\right)\left\langle\varphi\left(A_{1}\right)\left|\sigma_{x}\right| \chi\left(-A_{1}\right)\right\rangle, \\
D=\left(B_{2} / 2\right)\left(\left\langle\varphi^{\uparrow}\left|\sigma_{z}\right| \varphi^{\uparrow}\right\rangle+\left\langle\chi^{\uparrow}\left|\sigma_{z}\right| \chi^{\uparrow}\right\rangle\right)-D_{2}, \\
B=\left(B_{2} / 2\right)\left(\left\langle\varphi^{\uparrow}\left|\sigma_{z}\right| \varphi^{\uparrow}\right\rangle-\left\langle\chi^{\uparrow}\left|\sigma_{z}\right| \chi^{\uparrow}\right\rangle\right), \\
E_{0}=\left(E_{+}+E_{-}\right) / 2,
\end{gathered}
$$

and can be calculated numerically by using Eq. (7).

The numerical results of $\Delta, v_{F}, D$, and $B$ are presented in Fig. 2. It is noted that $|D|$ must be less than $|B|$, otherwise the energy gap will disappear, and all discussions in the following will not be valid. The $\Delta$ terms play a role of mass term in $2+1$ Dirac equations.

In the large $L$ limit,

$$
v_{F}=\left(A_{2} / \hbar\right) \sqrt{1-D_{1}^{2} / B_{1}^{2}} .
$$

The dispersion relation is given by

$$
\varepsilon_{c / v}(k)= \pm v_{F} \hbar k
$$

for small $k$. As a result, the energy gap closes at $k=0$. The two massless Dirac cones are located near the top and bottom surfaces, respectively, as expected in a 3D topological insulator.

In the small $L$ limit,

$$
v_{F}=A_{2} / \hbar
$$

and
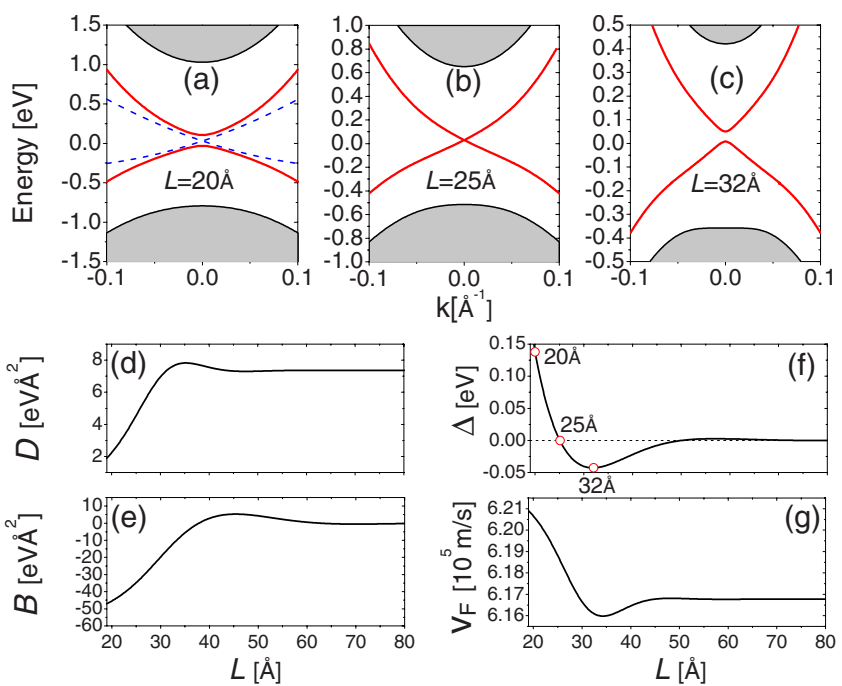

FIG. 2. (Color online) $[(\mathrm{a})-(\mathrm{c})]$ Twofold degenerate $\left(\tau_{z}= \pm 1\right)$ energy spectra of surface states for thicknesses $L=20,25,32 \AA$ (solid lines), and $L \rightarrow \infty$ (dash lines). The gray area corresponds to the bulk states. The energy spectra are obtained by solving $H\left(k,-i \partial_{z}\right) \Psi(z)=E \Psi(z)$ under the boundary conditions $\Psi(z= \pm L / 2)=0$. Please note that the scales of energy axis in (a)-(c) are different. The model parameters are adopted from Ref. 9: $M=0.28 \mathrm{eV}, A_{1}=2.2 \mathrm{eV} \mathrm{\AA ,} A_{2}=4.1 \mathrm{eV} \AA$, $B_{1}=10 \mathrm{eV} \mathrm{\AA} \AA^{2}, B_{2}=56.6 \mathrm{eV} \AA^{2}, C=-0.0068 \mathrm{eV}, D_{1}=1.3 \mathrm{eV} \AA^{2}$, and $D_{2}=19.6 \mathrm{eV}^{2}$. [(d)-(g)] The parameters for the new effective model $H_{\text {eff: }}: D, B$, the energy gap $\Delta$, and the Fermi velocity $v_{F}$ vs $L$.

$$
\Delta=2 B_{1} \pi^{2} / L^{2} .
$$

The ratio of the velocity between the two limits is

$$
\eta=1 / \sqrt{1-D_{1}^{2} / B_{1}^{2}} \text {. }
$$

It is noted that the velocity and energy gap for an ultrathin film are enhanced for a thinner film.

\section{ENERGY GAP AND k-DEPENDENT SPIN CONFIGURATION}

The opening of energy gap for the Dirac fermions is expected as a result of quantum tunneling between the surface states on the top and bottom surfaces. When the thickness of the ultrathin film is comparable with the decay length of the surface states into the bulk, the wave functions of the top and bottom surface states have a spatial overlap, which leads to an energy gap at the $\Gamma$ point, analogous to the splitting of the bound and antibound orbitals in a double-well potential. The dispersion relations of the surface states are plotted in Figs. 2(a)-2(c) for several thicknesses. A massless dispersion is obtained for the large $L$ limit as expected. For a finite thickness, the energy gap at $k=0$ is a function of the thickness $L$ and decays quickly with $L$ [see Fig. 2(f)]. It is noticed that the gap $\Delta$ even changes its sign at certain values of $L$. For instance, for the present case, at about integer times of $25 \AA$. Correspondingly, the velocity of the Dirac fermions is also thickness dependent, which is enhanced for a small $L$. 
(a) $\Delta / B<0$

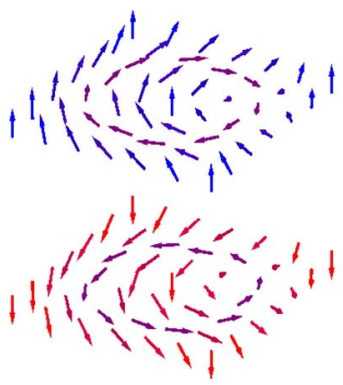

(b) $\Delta / B>0$
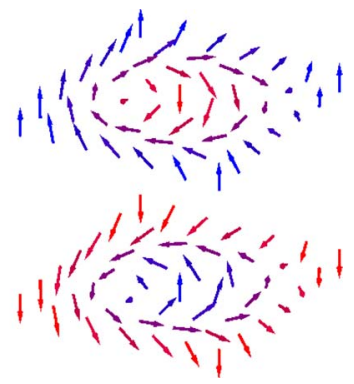

FIG. 3. (Color online) Schematic illustration of the k-dependent spin configurations in the conduction band (top) and valence band (bottom) in Dirac hyperbola $\tau_{z}=+1$ when (a) $\Delta / B<0$ and (b) $\Delta / B>0$. The center and corners of each panel correspond to that $k=0$ and $k$ is large enough, respectively.

Strictly speaking, for an ultrathin film, these so-called "surface states" emerge in the entire film. Nevertheless, they always lie in the bulk energy gap and can thus be distinguished from those bulk originated quantum well states.

From the solution to Eq. (10), it is obvious that the spin vectors in each band take a k-dependent spin configuration, in the neighborhood of the $\Gamma$ point determined by the gap parameter $\Delta$, and at large $k$ determined by the term $B \sigma_{z} k^{2}$, as schematically illustrated in Fig. 3. As the two Dirac hyperbolas are time-reversal copy of each other, it is obvious that they have just the opposite $\mathbf{k}$-dependent spin configurations, as can be seen from the $\tau_{z}$ dependence of $h_{\tau_{z}}(k)$ in Eq. (11).

\section{ORBITAL MAGNETIC MOMENT AND BERRY CURVATURES}

The opposite k-dependent spin configurations result in opposite physical properties of the surface Bloch electrons in the two Dirac hyperbolas, including the orbital magnetic moments and Berry curvatures, as shown in the context of graphene. ${ }^{20}$ These properties make possible the manipulation of spin dynamics by electric and magnetic fields in the thinfilm topological insulator.

Orbital magnetic moment arises from a self-rotating motion of the surface Bloch electron and is defined as

$$
m(\mathbf{k})=-i \frac{e}{2 \hbar}\left\langle\nabla_{k} u(\mathbf{k})\left|\times\left[h_{\tau_{z}}-\varepsilon(k)\right]\right| \nabla_{k} u(\mathbf{k})\right\rangle \cdot \hat{z}
$$

where $\varepsilon(\mathbf{k})$ and $u(\mathbf{k})$ are the dispersion and eigenstates of $h_{\tau}$, respectively. ${ }^{21,22}$ By putting Eqs. (12) and (13) into Eq. (20), we find the conduction and valence bands for each $h_{\tau_{z}}$ have the same orbital angular momentum

$$
m(k)=-\tau_{z} \frac{|e|}{\hbar} \frac{\hbar^{2} v_{F}^{2}\left(\Delta / 2+B k^{2}\right)}{2\left[\left(\Delta / 2-B k^{2}\right)^{2}+\hbar^{2} v_{F}^{2} k^{2}\right]} .
$$

At the $\Gamma$ point, the two degenerate Dirac hyperbolas acquire opposite orbital moments, which add to the spin magnetic moment.

The k-dependent spin configuration also results in a gauge field in the crystal momentum space, known as the Berry curvature
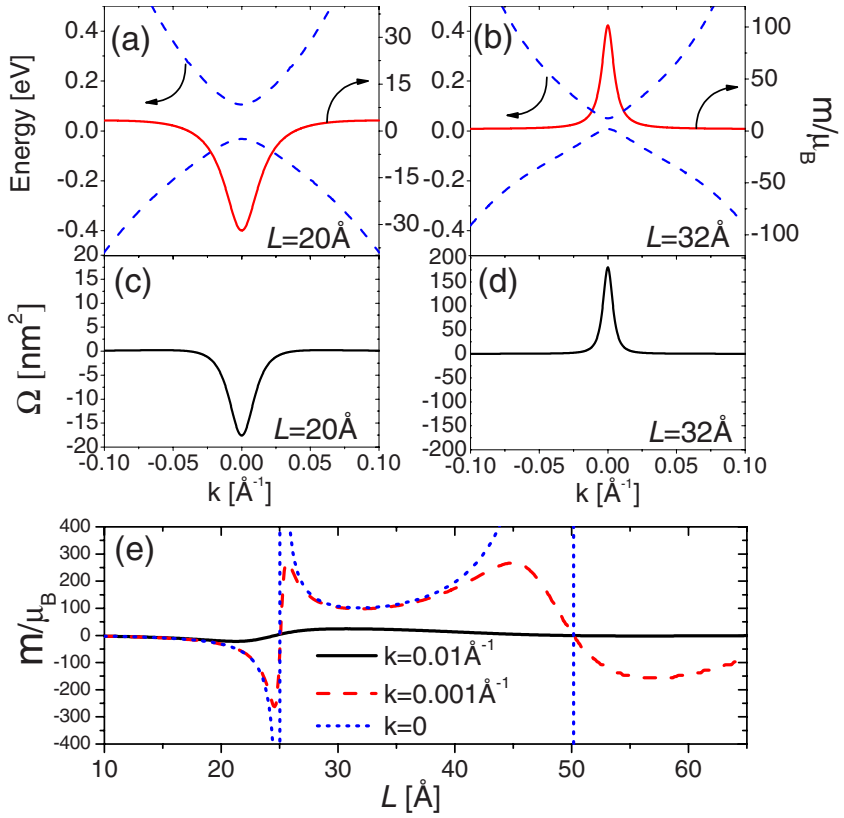

FIG. 4. (Color online) [(a) and (b)] Energy spectra of surface states (dash lines) and orbital angular moment $m$ of the conduction bands (solid lines) as functions of $k$ for $L=20$ and $32 \AA$. [(c) and (d)] Berry curvature $\Omega(k)$ of the conduction band. (e) Orbital angular moment $m$ of the conduction band as a function of thickness $L$ for $k=0.01,0.001$, and $0 \AA^{-1}$. The singularities occur at where $\Delta$ changes sign. Only results for the hyperbola $\tau_{z}=+1$ are shown. The results for $\tau_{z}=-1$ are right opposite to those for $\tau_{z}=+1$.

$$
\Omega(\mathbf{k})=\hat{z} \cdot \nabla_{\mathbf{k}} \times\left\langle u(\mathbf{k})\left|i \nabla_{k}\right| u(\mathbf{k})\right\rangle .
$$

In an in-plane electric field, electrons acquire an anomalous transverse velocity proportional to the Berry curvature, giving rise to the Hall effect. In the conduction bands, the Berry curvature distribution near the $\Gamma$ point is

$$
\Omega(k)=-\tau_{z} \frac{\hbar^{2} v_{F}^{2}\left(\Delta / 2+B k^{2}\right)}{2\left[\left(\Delta / 2-B k^{2}\right)^{2}+\hbar^{2} v_{F}^{2} k^{2}\right]^{3 / 2}} .
$$

The Berry curvature in the valence bands is right opposite to that of in the conduction bands. There are opposite distributions of the Berry curvature in the two Dirac hyperbolas. The in-plane electric field can therefore drive the spin-up and spin-down electrons toward the opposite transverse edges of the thin film, which is a surface spin Hall effect. Numerical results for the orital angular mometum and the Berry curvature as functions of $k$ and thickness $L$ are presented in Fig. 4.

\section{SPIN HALL EFFECT AND TOPOLOGICAL QUANTUM PHASE TRANSITION}

The $\Delta$ term in our model plays a role as the magnetization in the massive Dirac model exploited to study the anomalous Hall effect. ${ }^{23}$ In principle, we can find the Hall conductance for each $h_{\tau_{z}}$. Note that $h_{\tau_{z}}$ in Eq. (11) can be explicitly written as 


$$
h_{\tau_{z}}=E_{0}-D k^{2}+\sum_{i=x, y, z} d_{i} \sigma_{i}
$$

where $\sigma_{i}$ are the Pauli matrices and the $\mathbf{d}(k)$ vectors

$$
d_{x}=\hbar v_{F} k_{y}, \quad d_{y}=-\hbar v_{F} k_{x}, \quad d_{z}=\tau_{z}\left(\frac{\Delta}{2}-B k^{2}\right) .
$$

For the $2 \times 2$ Hamiltonian in terms of the $\mathbf{d}(k)$ vectors and Pauli matrices, the Kubo formula for the Hall conductance can be generally expressed as ${ }^{24,25}$

$$
\sigma_{x y}=\frac{e^{2}}{2 \hbar} \int \frac{d^{2} \mathbf{k}}{(2 \pi)^{2}} \frac{\left(f_{k, c}-f_{k, v}\right)}{d^{3}} \epsilon_{\alpha \beta \gamma} \frac{\partial d_{a}}{\partial k_{x}} \frac{\partial d_{\beta}}{\partial k_{y}} d_{\gamma},
$$

where $d$ is the norm of $\left(d_{x}, d_{y}, d_{z}\right), f_{k, c / v}$ $=1 /\left\{\exp \left\{\left[\varepsilon_{c / v}(k)-\mu\right] / k_{B} T\right\}+1\right\}$ the Fermi distribution function of the conduction $(c)$ and valence $(v)$ bands with $\mu$ the chemical potential, $k_{B}$ the Boltzmann constant, and $T$ the temperature.

At zero temperature and when the chemical potential $\mu$ lies between $\left(-\frac{|\Delta|}{2}, \frac{|\Delta|}{2}\right)$, the Fermi functions reduce to $f_{k, c}=0$ and $f_{k, v}=1$. By substituting Eq. (25) into Eq. (26) we arrive at

$$
\sigma_{x y}\left(0, \tau_{z}\right)=-\tau_{z} \frac{e^{2}}{4 h} \int_{0}^{\infty} d\left(k^{2}\right) \frac{\left(\hbar v_{F}\right)^{2}\left(\frac{\Delta}{2}+B k^{2}\right)}{\left[\left(\hbar v_{F}\right)^{2} k^{2}+\left(\frac{\Delta}{2}-B k^{2}\right)^{2}\right]^{3 / 2}} .
$$

Note that by comparing above equation with Eq. (23), we can write the Hall conductance in the form of the Berry curvature of the conduction band,

$$
\sigma_{x y}\left(0, \tau_{z}\right)=\frac{e^{2}}{\hbar} \int \frac{d^{2} \mathbf{k}}{(2 \pi)^{2}}\left(f_{k, v}-f_{k, c}\right) \Omega(k) .
$$

By defining

$$
\cos \theta=\frac{\left(\frac{\Delta}{2}-B k^{2}\right)}{\sqrt{\left(\hbar v_{F}\right)^{2} k^{2}+\left(\frac{\Delta}{2}-B k^{2}\right)^{2}}},
$$

Eq. (27) can be transformed into

$$
\sigma_{x y}\left(0, \tau_{z}\right)=\tau_{z} \frac{e^{2}}{2 h} \int_{0}^{\infty} d\left(k^{2}\right) \frac{\partial(\cos \theta)}{\partial\left(k^{2}\right)} .
$$

The values of $\cos \theta$ at $k=0$ and $k \rightarrow \infty$ only depend on the signs of $B$ and $\Delta$, respectively. As a result, in the insulating regime $-\frac{|\Delta|}{2} \leq \mu \leq \frac{|\Delta|}{2}$, we find that the anomalous Hall conductance for each hyperbola has the form

$$
\sigma_{x y}\left(0, \tau_{z}\right)=-\tau_{z} \frac{e^{2}}{2 h}[\operatorname{sgn}(\Delta)+\operatorname{sgn}(B)],
$$

where $\frac{\tau_{z}}{2}[\operatorname{sgn}(\Delta)+\operatorname{sgn}(B)]$ is the Chern number of the valence bands. ${ }^{26}$ It is a well-known result in the field theory that the Hall conductance of the massive Dirac fermions is a half of quanta $e^{2} / h, \sigma_{x y}=-\tau_{z} \frac{e^{2}}{2 h} \operatorname{sgn}(\Delta)$ (if $\left.B=0\right)$ (see Ref. 27).

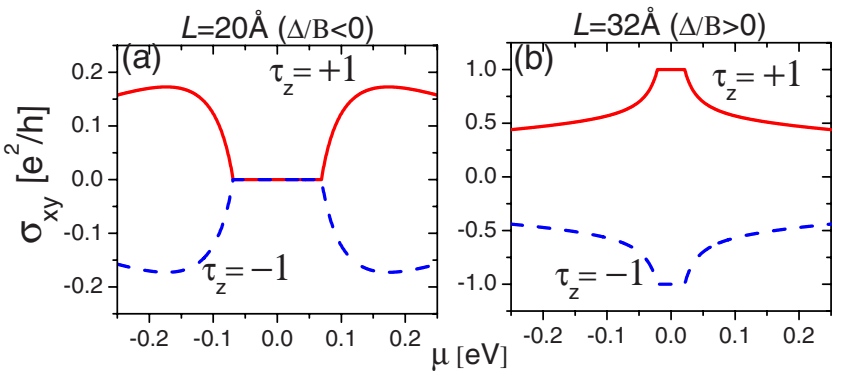

FIG. 5. (Color online) The hyperbola-dependent Hall conductance vs Fermi level $\mu$ for (a) $L=20 \AA$ and (b) $32 \AA$, respectively.

Our result demonstrates that a nonzero quadratic term in $k(B \neq 0)$ will give a reasonable result since a half-quantized Hall conductance is not possible for a noninteracting system. For small $L$, the parameter $B$ is always negative and $\Delta$ changes its sign accompanying a gap close-and-reopen while the thickness of the thin film increases [Fig. 2(f)]. The sign change in $\Delta$ flips the k-dependent spin configuration near the $\Gamma$ point and results in a jump $\tau_{z}$ in the Chern number, ${ }^{28,29}$ i.e., a topological quantum phase transition as discussed in quantum spin Hall effect in $\mathrm{HgTe} / \mathrm{CdTe}$ quantum well. ${ }^{30}$ In the ultrathin limit, $\Delta>0$ and the Hall conductance is zero, while $\Delta<0$ at a larger thickness and the Hall conductance $\sigma_{x y}\left(0, \tau_{z}\right)=\tau_{z}\left(e^{2} / h\right)$, as shown in Fig. 5. By the edge-bulk correspondence, ${ }^{31}$ gapless helical edge states shall appear accompanying such a transition. This result is indeed supported by the solutions of the differential equation of $h_{ \pm}(k)$ in a geometry of a semi-infinite plane, in which there exists a gapless edge state only when $\Delta / B>0$, and the sign of the Hall conductance can be justified from the chirality of the edge states.

When the Fermi level $\mu$ lies in the electron band, according to Eq. (26)

$$
\sigma_{x y}\left(\mu>\frac{|\Delta|}{2}, \tau_{z}\right)=\sigma_{x y}\left(0, \tau_{z}\right)+\tau_{z} \sigma_{x y}^{+},
$$

where at zero temperature,

$$
\sigma_{x y}^{+}=\frac{e^{2}}{4 h} \int_{0}^{k_{F}} d\left(k^{2}\right) \frac{A^{2}\left(\frac{\Delta}{2}+B k^{2}\right)}{\left[A^{2} k^{2}+\left(\frac{\Delta}{2}-B k^{2}\right)^{2}\right]^{3 / 2}} .
$$

Near the $\Gamma$ point, the $B$ term in the electron-band dispersion can be neglected, so that $\varepsilon_{c}^{2} \approx\left(\hbar v_{F}\right) k^{2}+\left(\frac{\Delta}{2}\right)^{2}$ and

$$
k_{F}^{2}=\left[\mu^{2}-\left(\frac{\Delta}{2}\right)^{2}\right] /\left(\hbar v_{F}\right)^{2} .
$$

After a straightforward calculation and Taylor expansion of the result up to the linear term of $\mu$, the Hall conductance is obtained when the Fermi surface is in the conduction bands,

$$
\sigma_{x y}\left(\mu>\frac{|\Delta|}{2}, \tau_{z}\right)=-\tau_{z} \frac{e^{2}}{2 h}\left[\operatorname{sgn}(B)+\operatorname{sgn}(\Delta)-\frac{8 \pi\left(\hbar v_{F}\right)^{2} \delta n}{\Delta|\Delta|}\right],
$$


where the Fermi level $\mu$ is replaced by $\delta n$, the carrier density near the band bottom, by using the relation $|\Delta|\left(\mu-\frac{|\Delta|}{2}\right) /\left(4 \pi \hbar^{2} v_{F}^{2}\right) \approx \delta n$, which is found by using the dispersion $\varepsilon_{c}$ and the same Taylor expansion approach. One can check that when the Fermi level lies in the valence bands, $\sigma_{x y}$ is the same as the above result due to the particle-hole symmetry.

As shown in Fig. 2(e), $B$ is negative for small $L$. For $\Delta>0$ where the intrinsic film is a trivial insulator, doping with electrons or holes can result in a metallic spin Hall effect, with the Hall conductance given by

$$
\sigma_{x y}\left(\mu>\frac{\Delta}{2}, \tau_{z}\right)=\tau_{z} \frac{e^{2}}{h} \frac{4 \pi}{\Delta^{2}}\left(\hbar v_{F}\right)^{2} \delta n,
$$

where $\delta n$ is the carrier density. In the two Dirac hyperbolas, the Hall conductance are opposite (see Fig. 5) and spin vectors near the band bottom point in the $+z$ and $-z$ directions, respectively. Thus net spin accumulations with out-of-plane polarization are expected on the two edges of the thin film. For $\Delta<0$, doping reduces the quantized Hall conductance and we find

$$
\sigma_{x y}\left(\mu>\frac{|\Delta|}{2}, \tau_{z}\right)=\tau_{z} \frac{e^{2}}{h}\left[1-\frac{4 \pi}{\Delta^{2}}\left(\hbar v_{F}\right)^{2} \delta n\right] .
$$

The Hall conductance as a function of the Fermi level $\mu$ are plotted in Fig. 5. Note that because the Hall conductances for the two hyperbolas are always equal in magnitude and opposite in sign, and the two hyperbolas have right opposite spin orientations, here the Hall conductances are referred to as the ordinary and quantum spin Hall effects.

\section{SPIN OPTICAL SELECTION RULE}

Since an energy gap is opened in the Dirac hyperbolas, interband transitions between the conduction and valence surface bands can be excited by optical field. In the two gapped Dirac hyperbolas being time reversal of each other, interband transition couples preferentially to right-handed $(\sigma+)$ or left-handed $(\sigma-)$ circular polarized light, as was first discovered in the context of graphene. ${ }^{32}$ In the thin-film topological insulator where the two Dirac hyperbolas are associated with opposite spin configurations (Fig. 3), such an optical transition selection rule is of significance since it allows the spin dynamics to be addressed by optical means. The interband couplings in the two hyperbolas to normally incident circular polarized lights can be studied by calculating the degree of circular polarization, defined as

$$
\eta(\mathbf{k}) \equiv \frac{\left|\pi_{c v}^{+}(\mathbf{k})\right|^{2}-\left|\pi_{c v}^{-}(\mathbf{k})\right|^{2}}{\left|\pi_{c v}^{+}(\mathbf{k})\right|^{2}+\left|\pi_{c v}^{-}(\mathbf{k})\right|^{2}}
$$

where \pm corresponding to $\sigma \pm$ lights, respectively, and the interband matrix element of the velocity operator is defined by

$$
\pi_{c v}^{ \pm}(\mathbf{k}) \equiv\left\langle u_{c}(\mathbf{k})\left|\frac{\partial h_{\tau_{z}}}{\partial k_{x}} \pm i \frac{\partial h_{\tau_{z}}}{\partial k_{y}}\right| u_{v}(\mathbf{k})\right\rangle,
$$

where $u_{c}(\mathbf{k})$ and $u_{v}(\mathbf{k})$ are the eigenstates for the conduction and valence bands of $h_{\tau_{z}}$, respectively, and have already been given in Eq. (13). By ignoring $B k^{2}$ terms near the $\Gamma$ point, one finds

$$
\left|\pi_{c v}^{ \pm}(k)\right|^{2} \simeq\left(\hbar v_{F}\right)^{2}\left(1 \pm \tau_{z} \cos \theta\right)^{2}
$$

where $\cos \theta=\Delta /\left[\varepsilon_{c}(k)-\varepsilon_{v}(k)\right]$ with $\varepsilon_{c}\left(\varepsilon_{v}\right)$ the dispersion of the conduction (valence) band of $h_{\tau_{z}}$ given in Eq. (12). Then the degree of polarization is found out for each $\tau_{z}$

$$
\eta(k)=\tau_{z} \frac{2 \cos \theta}{1+\cos ^{2} \theta} .
$$

Near the Dirac hyperbola center where $\varepsilon_{c}(k)-\varepsilon_{v}(k) \simeq|\Delta|$, $\sigma+(\sigma-)$ light couples only to the Dirac hyperbola $\tau_{z}=\operatorname{sgn}(\Delta)\left[\tau_{z}=-\operatorname{sgn}(\Delta)\right]$, causing transition between the spin-down (up) valence state and the spin-up (down) conduction state. This is in sharp contrast to the spin optical transition selection rule in conventional semiconductor, which leaves the spin part of the wave function unchanged. This spin optical transition selection rule makes possible optical injection of spins in the thin film. For example, with bandedge optical excitation by $\sigma+$ circularly polarized light, spin-up electrons in the conduction band and spin-up holes (by convention, a spin-up hole refers to an empty spin-down valence state) in the valence band are created in the Dirac hyperbola $\tau_{z}=\operatorname{sgn}(\Delta)$, which can be separated by an in-plane electric field to prevent the radiative recombination.

\section{CONCLUSIONS}

In this paper, we derive an effective model for an ultrathin film of topological insulator, in which tunneling between the surface states on the top and bottom surfaces opens a finite gap in the Dirac cone centered at the $\Gamma$ point $(k=0)$. The low-lying physics of the ultrathin film can be described as two degenerate massive Dirac hyperbolas, which form timereversal copy of each other. Each massive band has a k-dependent spin configuration: one near the $\Gamma$ point determined by the energy gap and the other at $k$ large enough determined by a spin-orbit coupling term quadratic in $k$. It is found that the energy gap oscillates with the thin-film thickness and changes sign at critical thicknesses. Across the transition points, the k-dependent spin configuration near the $\Gamma$ point is flipped while those at large $k$ remains unchanged, leading to a topological quantum phase transition where the Chern numbers of the surface bands change [Eq. (31)]. In the two Dirac hyperbolas of opposite spin configurations, the $\mathbf{k}$-dependent spin structure results in a distribution of orbital magnetic moment and Berry curvature with opposite signs. In doped metallic regime, the Berry curvature drives the spin Hall effect of the extra carriers, which leads to net spin accumulations on the thin-film edges. We also discover a spindependent optical transition selection rule, which allows optical injection of spins by circular polarized lights into the thin film.

Note added. After posting this paper on arXiv, we learnt about the works by Liu et al. ${ }^{33}$ and Linder et al. ${ }^{34}$ in which the similar finite-size effect of the surface states was studied. 


\section{ACKNOWLEDGMENTS}

This work was supported by the Research Grant Council of Hong Kong under Grants No. HKU 7037/08P and No.
HKU 10/CRF/08. Q.N. was supported in part by DoE, NSF, the Welch foundation F-1255, and the Texas Advanced Research Program.
${ }^{1}$ L. Fu, C. L. Kane, and E. J. Mele, Phys. Rev. Lett. 98, 106803 (2007).

${ }^{2}$ J. E. Moore and L. Balents, Phys. Rev. B 75, 121306(R) (2007).

${ }^{3}$ S. Murakami, New J. Phys. 9, 356 (2007).

${ }^{4}$ J. C. Y. Teo, L. Fu, and C. L. Kane, Phys. Rev. B 78, 045426 (2008).

${ }^{5}$ D. Hsieh, D. Qian, L. Wray, Y. Xia, Y. S. Hor, R. J. Cava, and M. Z. Hasan, Nature (London) 452, 970 (2008).

${ }^{6}$ D. Hsieh, Y. Xia, L. Wray, D. Qian, A. Pal, J. H. Dil, J. Osterwalder, F. Meier, G. Bihlmayer, C. L. Kane, Y. S. Hor, R. J. Cava, and M. Z. Hasan, Science 323, 919 (2009).

${ }^{7}$ A. Nishide, A. A. Taskin, Y. Takeichi, T. Okuda, A. Kakizaki, T. Hirahara, K. Nakatsuji, F. Komori, Y. Ando, and I. Matsuda, arXiv:0902.2251 (unpublished).

${ }^{8}$ Y. Xia, D. Qian, D. Hsieh, L. Wray, A. Pal, H. Lin, A. Bansil, D. Grauer, Y. S. Hor, R. J. Cava, and M. Z. Hasan, Nat. Phys. 5, 398 (2009).

${ }^{9}$ H. J. Zhang, C. X. Liu, X. L. Qi, X. Dai, Z. Fang, and S. C. Zhang, Nat. Phys. 5, 438 (2009).

${ }^{10}$ D. Hsieh, Y. Xia, D. Qian, L. Wray, J. H. Dil, F. Meier, J. Osterwalder, L. Patthey, J. G. Checkelsky, N. P. Ong, A. V. Fedorov, H. Lin, A. Bansil, D. Grauer, Y. S. Hor, R. J. Cava, and M. Z. Hasan, Nature (London) 460, 1101 (2009).

${ }^{11}$ Y. L. Chen, J. G. Analytis, J.-H. Chu, Z. K. Liu, S.-K. Mo, X. L. Qi, H. J. Zhang, D. H. Lu, X. Dai, Z. Fang, S. C. Zhang, I. R. Fisher, Z. Hussain, and Z.-X. Shen, Science 325, 178 (2009).

${ }^{12}$ X. L. Qi, T. L. Hughes, and S. C. Zhang, Phys. Rev. B 78, 195424 (2008).

${ }^{13}$ L. Fu and C. L. Kane, Phys. Rev. Lett. 100, 096407 (2008).

${ }^{14}$ J. Nilsson, A. R. Akhmerov, and C. W. J. Beenakker, Phys. Rev. Lett. 101, 120403 (2008).

${ }^{15}$ L. Fu and C. L. Kane, Phys. Rev. Lett. 102, 216403 (2009).

${ }^{16}$ A. R. Akhmerov, J. Nilsson, and C. W. J. Beenakker, Phys. Rev.
Lett. 102, 216404 (2009).

${ }^{17}$ Y. Tanaka, T. Yokoyama, and N. Nagaosa, Phys. Rev. Lett. 103, 107002 (2009).

${ }^{18}$ K. T. Law, P. A. Lee, and T. K. Ng, Phys. Rev. Lett. 103, 237001 (2009).

${ }^{19}$ B. Zhou, H. Z. Lu, R. L. Chu, S. Q. Shen, and Q. Niu, Phys. Rev. Lett. 101, 246807 (2008).

${ }^{20}$ D. Xiao, W. Yao, and Q. Niu, Phys. Rev. Lett. 99, 236809 (2007).

${ }^{21}$ M. C. Chang and Q. Niu, J. Phys.: Condens. Matter 20, 193202 (2008).

${ }^{22}$ D. Xiao, M. C. Chang, and Q. Niu, arXiv:0907.2021 (unpublished).

${ }^{23}$ N. A. Sinitsyn, A. H. MacDonald, T. Jungwirth, V. K. Dugaev, and J. Sinova, Phys. Rev. B 75, 045315 (2007).

${ }^{24}$ X. L. Qi, Y. S. Wu, and S. C. Zhang, Phys. Rev. B 74, 085308 (2006).

${ }^{25}$ B. Zhou, L. Ren, and S. Q. Shen, Phys. Rev. B 73, 165303 (2006).

${ }^{26}$ D. J. Thouless, M. Kohmoto, M. P. Nightingale, and M. den Nijs, Phys. Rev. Lett. 49, 405 (1982).

${ }^{27}$ A. N. Redlich, Phys. Rev. D 29, 2366 (1984).

${ }^{28}$ S. Q. Shen, Phys. Rev. B 70, 081311(R) (2004).

${ }^{29}$ B. Zhou, C. X. Liu, and S. Q. Shen, EPL 79, 47010 (2007).

${ }^{30}$ B. A. Bernevig, T. L. Hughes, and S. C. Zhang, Science 314, 1757 (2006).

${ }^{31}$ Y. Hatsugai, Phys. Rev. Lett. 71, 3697 (1993).

${ }^{32}$ W. Yao, D. Xiao, and Q. Niu, Phys. Rev. B 77, 235406 (2008).

${ }^{33}$ C. X. Liu, H. Zhang, B. Yan, X. L. Qi, T. Frauenheim, X. Dai, Z. Fang, and S. C. Zhang, Phys. Rev. B 81, 041307(R) (2010).

${ }^{34}$ J. Linder, T. Yokoyama, and A. Sudbø, Phys. Rev. B 80, 205401 (2009). 